\title{
Simple, time saving pulsed-field gel electrophoresis protocol for the typing of Stenotrophomonas maltophilia
}

\begin{abstract}
We developed a time-saving and cost-efficient Pulsed Field Gel Electrophoresis (PFGE) method for the typing of Stenotrophomonas maltophilia by modifying the conventional procedures. Our modifications related to the cell suspension preparation, lysis of bacterial cells in plugs, washing steps, and consumption of restriction enzyme. Although few rapid PFGE protocols on Gram-negative bacteria are available, the use of comparatively large amounts of costly reagents prompted us to look for other alternative. Hence, by considering the speed, simplicity, and relatively low cost, the modified protocol may be of more practical value than other established protocols in investigating S. maltophilia nosocomial outbreaks.
\end{abstract}

Keyword: Stenotrophomonas maltophilia; Pulsed Field Gel Electrophoresis; PFGE; Timesaving; Cost efficient 